\title{
Liquid Metal Flow with Heat Transfer in a Cold Crucible Confined by a Free Surface and a Solidification Front
}

\author{
Tsutomu TANAKA, Koichi KURITA and Atsuhiko KURODA ${ }^{1)}$ \\ Iron and Steel Research Laboratories, Sumitomo Metal Industries, Ltd., Sunayama, Hasaki, Kashima-gun, Ibaraki-ken, 314-02 \\ Japan. 1) Advanced Technology Research Laboratories, Sumitomo Metal Industries, Ltd., Fuso-cho, Amagasaki, \\ Hyogo-ken, 660 Japan
}

(Received on July 30, 1991; accepted in final form on October 25, 1991)

\begin{abstract}
An advanced mathematical model for the fluid flow coupled with heat transfer confined by a free surface and a solidification front in a cold crucible was developed. Validity of the model was confirmed through measurements of a solidification front, surface velocity of the melt and surface temperatures.

Effects of operation parameters, such as coil current, dome height and casting velocity, on the fields of velocity and temperature were investigated.

Generally, two kinds of recirculation flow are expected to appear in the melt. They make a collision slightly above the contact point between the melt and the crucible wall. An appropriate geometrical configuration of the dome height and the position of a coil exists to promote melting of scraps fed onto dome.

A titanium ingot consisting of completely melted scraps was continuously cast aided by the proposed operation obtained through the numerical calculation.
\end{abstract}

KEY WORDS: cold crucible; electromagnetism; liquid metal; continuous casting; solidification front; fluid flow; free surface.

\section{Introduction}

In a cold crucible, thermal energy is transmitted into the bulk of a charge in a contactless way and the contact between the liquid metal and the curcible wall is limited by taking advantage of pinch force induced in the melt. As a result, ingot is hardly polluted by the crucible wall during continuous casting of reactive materials with high melting temperature, such as titanium ${ }^{1)}$ or $\mathrm{Si}$ semiconductor. ${ }^{2)}$ Theoretical studies as well as experimental ones on the liquid metal flow coupled with the heat transfer of a charge in the crucible are important for the optimization of the design of the crucible and the choice of the appropriate operation condition of casting.

Various kinds of difficulties accompanies with the development of a mathematical model capable of describing the metal flow coupled with heat transfer of a charge. They are the following. 1) Geometry of the crucible is of three dimension. 2) Fields of electromagnetics, velocity and temperature are mutually interacted. As a result, two kinds of free boundaries exist associated with the free surface of the melt and the solidification front. 3) Since a long range type of an interaction exists in the electromagnetic field and the field is a periodic function of time, an integral equation with complex variable appears in the governing equation.

There are a few previous studies in this kind of field. B. Maestrali et al. $^{3)}$ developed a model describing the liquid metal flow coupled with heat transfer on the assumption of weak interaction for the coupling between the electromagnetic field and the melt shape. The model has a problem in the treatment of the melt shape as the input to the model.

The aims of the present study are the following. 1) Development of an advanced mathematical model capable of describing liquid metal flow with heat transfer in a domain confined by a free surface and a solidification front. 2) Making a better understanding with respect to the effects of various operation parameters, such as melt volume, coil current, heat loss and casting velocity, on the melt shape, solidification front and the fields of velocity and temperature. 3) Examination of continuous casting by making use of a cold crucible aided by the information obtained through the model.

\section{Mathematical Model}

Schematic structure of the whole mathematical model is shown in Fig. 1. The model comprises three kinds of submodels. They are electromagnetic field model, fluid flow model and heat transfer model. Moreover, the electromagnetic field model consists of two kinds of submodels. These models are mutually coupled through the interactions of electromagnetics, fluid dynamics and heat transfer. The whole model has two kinds of free boundaries, namely a free surface and a solidification front, which must be determined through the interaction among the models. 


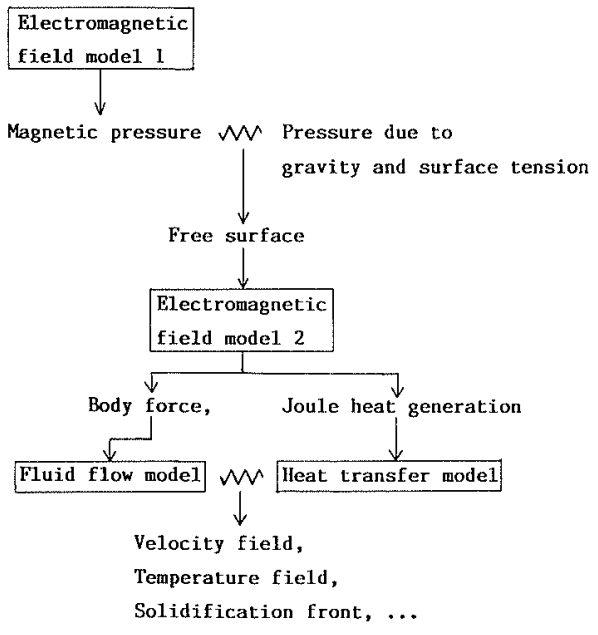

Fig. 1. Relation among mathematical models. Symbols $M$ and $\rightarrow$ mean the existence of an interaction and the direction of data flow, respectively.

\subsection{Electromagnetic Field Model 1}

Details of the electromagnetic field moedl 1 are described in the reference. ${ }^{4)}$ It has the following characteristics. 1) Because of the presence of various number of slits along the vertical direction of the crucible wall, the structure of the crucible is strictly of three dimension. However, by introducing two kinds of model parameters representing the continuity of the current density and the magnitude of the electromagnetic interaction on the slit part of the crucible, three dimensional model reduces to two dimensional axisymmetric one without loss of accuracy. 2) An integration of the magnetic field along the depth of the conductor can be written analytically by applying the Lorentz gauge to the Maxwell's equations. As a result, the model further reduces to a sheet model composed of an ensemble of filmy ribbons. 3) The shape of the melt is obtained through the force balance among electromagnetic pressure, gravitational force and surface tension. This method is valid for the values of shield parameter $R_{\omega}=\mu \sigma \omega R^{2}$ larger than $10 .{ }^{5)}$ Usually, this condition holds in a typical operation of a cold crucible.

Hence, the consideration of the coupling between the electromagnetic field and the field of flow or that of temperature is unnecessary in the numerical calculation of the melt shape when the temperature of a dome is high enough to be a liquid.

\subsection{Electromagnetic Field Model 2}

In the electromagnetic field model 2 , the electromagnetic body force and the joule heat generation used in the calculation of fluid flow and temperature in a charge is obtained on the basis that the melt is the same shape that was calculated through the electromagnetic field model 1. The effect of the velocity of melt on the induced current is negligibly small, since the magnetic Reynolds number, $R_{m}=\mu \sigma v R$, is $5 \times 10^{-3}$, which is shown in the following section. Hence,

$$
\boldsymbol{j}=-\sigma\left(\operatorname{grad} \phi+\frac{\partial \boldsymbol{A}}{\partial t}-\boldsymbol{v} \times \boldsymbol{B}\right) \fallingdotseq-\sigma\left(\operatorname{grad} \phi+\frac{\partial \boldsymbol{A}}{\partial t}\right)
$$

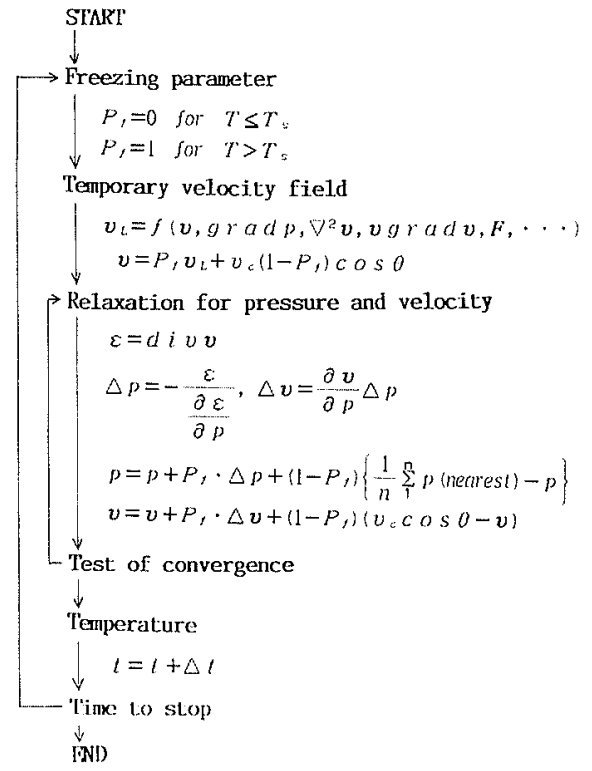

Fig. 2. Flow chart in the calculation of velocity field coupled with heat transfer during solidification.

In the analysis of the electromagnetic field, it is necessary to obtain the field in a charge, since the distribution of the electromagnetic body force induces the melt flow. Therefore, a model composed of volume elements for the charge and sheet elements for the crucible as well as the coil is adopted. The governing equations of the electromagnetic field in the former and the latter elements are obtained through the application of Coulomb gauge and the Lorentz gauge to the Maxwell's equation, respectively. For example, vector potential is represented by the following integral expressions depending on the species of conductor.

$$
\begin{aligned}
& A=\frac{\mu}{4 \pi} \int \frac{j}{r} \exp \{(i-1) r / \delta\} d V \quad \text { crucible and coil ... } \\
& \boldsymbol{A}=\frac{\mu}{4 \pi} \int \frac{\boldsymbol{j}}{r} d V \quad \text { charge }
\end{aligned}
$$

Equation (1) in conjunction with the Eq. (2) or Eq. (3) constitutes the governing integral equation with the unknown variable of the current density $j$ if the scalar potential $\phi$ is known. The scalar potential on the coil is the homogeneous voltage applied on the coil edge and is given as the boundary condition. The scalar potentials except for the coil are given by the continuity condition of the current density. Gauss-Legendre integration formula with four divisions of integration points was adopted in the calculation to avoid the divergence in the self-integration when $r=0$ as well as to improve the accuracy of integration.

\subsection{Fluid Flow Model}

The free boundary of the solidification front is to be determined together with the fields of velocity and temperature through the fluid flow model coupled with heat transfer. The calculation scheme is shown in Fig. 2. The characteristics of the scheme lie in the introduction of a freezing parameter $P_{f}$ and making use of freezing parameter during the relaxation for the velocity and the 
pressure.

Calculation scheme is the following. First of all, the freezing parameter is estimated according to the distribution of the temperature of a charge in such a way that the parameter is zero where the temperature is lower than that of solidus and it is one where the temperature is higher. Secondary, the temporary velocity is evaluated, followed by the replacement of the velocity to be equal to the casting one whose freezing parameter is zero. Thirdly, the residual in the divergence of the velocity is relaxed by making use of NewtonRaphson method. In this case, the pressure and the velocity are calculated so as to equal to the mean value with respect to the nearest neighbor cells and the withdrawal velocity of the ingot, respectively, in the cell where the freezing parameter is zero. This operation is conducted followed by the calculation of temperature with the advance in time.

Because of the presence of the free surface in the calculation domain, boundary fitted general coordinate is to be used. The governing equation of continuity and those of motion in non-orthogonal curvilinear coordinate for an incompressible Newtonian fluid are written by Eqs. (4) and (5), respectively. ${ }^{6)}$

$$
\begin{array}{r}
\frac{1}{\sqrt{g}} \frac{\partial}{\partial \xi^{k}}\left\{\frac{\sqrt{g}}{\sqrt{g_{k k}}} v(k)\right\}=0 \quad \ldots \ldots \ldots \ldots \ldots . . . .(4) \\
\frac{\partial v(i)}{\partial t}+\frac{\sqrt{g_{\overline{i i}}}}{\sqrt{g_{k F}}} v(k) \frac{\partial}{\partial \xi^{k}} \frac{v(i)}{\sqrt{g_{\overline{i i}}}}=-\frac{1}{\rho} g^{k i} \sqrt{g_{\bar{i}}} \frac{\partial p}{\partial \xi^{k}} e(i) \\
+v \frac{1}{\sqrt{g}} \frac{\partial}{\partial \xi^{l}}\left\{\sqrt{g} g^{k l} \frac{\partial v(i)}{\partial \xi^{k}}\right\}+\frac{1}{2 \rho}\left\{\operatorname{Re}\left(j \times B^{*}\right)\right\}(i)
\end{array}
$$

where, $g_{k l}=\frac{\partial x_{i}}{\partial \xi_{k}} \frac{\partial x_{i}}{\partial \xi_{l}}, g^{k l}=\frac{\text { cofactor } g_{k l}}{g}, g=\operatorname{det}\left(g_{k l}\right)$.

The last term in the right hand side of Eq. (5) is the time averaged electromagnetic body force and is given as the result of the electromagnetic field model 2 .

The flow of the melt caused by electromagnetic force is turbulent. However, an ad hoc equation was not used in the model and the laminar viscosity was applied to the melt. The temperature dependence of viscosity was approximated by pseudo Heaviside function written by Eq. (6), representing the increase in the viscosity during solidification.

$$
\nu=\frac{v_{S}}{\pi} \times\left\{\frac{\pi}{2}-\tan ^{-1}\left(\frac{T-T_{M}}{P_{v}}\right)\right\}+v_{L}
$$

\subsection{Heat Transfer Model}

The governing equation of heat transfer in general coordinate is given by Eq. (7).

$$
\begin{aligned}
\frac{d H}{d T} & \frac{\partial T}{\partial t}+\frac{\sqrt{g_{\overline{i i}}}}{\sqrt{g_{k K}}} v(k) \frac{\partial}{\partial \xi^{k}} \frac{T}{\sqrt{g_{\overline{i i}}}} \frac{d H}{d T} \\
= & \frac{\lambda}{\rho} \frac{1}{\sqrt{g}} \frac{\partial}{\partial \xi^{l}}\left\{\sqrt{g} g^{k l} \frac{\partial T}{\partial \xi^{k}}\right\} \\
& +\frac{1}{\rho}\left\{\frac{1}{2 \sigma} \operatorname{Re}\left(j \cdot j^{*}\right)+Q_{m}+Q_{l}\right\} \ldots
\end{aligned}
$$

The temperature dependence of enthalpy was approximately written by pseudo Delta function. That is,

$$
\frac{d H}{d T}=C_{p}+\frac{\Delta H}{\pi} \times \frac{\varepsilon_{H}}{\left(T-T_{M}\right)^{2}+\varepsilon_{H}^{2}}
$$

where, $\varepsilon_{H}=\frac{\Delta H}{\pi C_{p}\left(P_{H}-1\right)}, P_{H}=\left.\frac{1}{C_{p}} \frac{d H}{d T}\right|_{T=T_{M}}$

The heat loss of the melt corresponding to the decrease in the melt temperature when a metallic raw material is added onto the dome was estimated through the assumptions of the homogeneity of the melt and the stationary state of the melt temperature during casting. Hence,

$$
Q_{m}=\frac{\dot{m}_{m}}{V_{p}}\left\{H\left(T_{m}\right)-H\left(\bar{T}_{p}\right)\right\}
$$

The enthalpy is obtained through the integration of Eq. (8) with respect to temperature, resulting,

$$
H(T)=C_{p} T+\frac{\Delta H}{\pi}\left(\tan ^{-1} \frac{T-T_{M}}{\varepsilon_{H}}+\tan ^{-1} \frac{T_{M}}{\varepsilon_{H}}\right) \ldots
$$

When the heat loss of the charge is written as that through the surface by the boundary condition depending on the temperatures of the charge and the surroundings, the amount of heat loss is likely to be overestimated unless the effect of the convection of the melt on the heat loss is accurately estimated. For the simplicity and the improvement of the accuracy of the calculation, the heat loss through the surface was mathematically described as that through the volume element on the nearest cell to the surface of the charge. In this case, the adiabatic boundary condition was applied to the temperature to avoid the double estimation of the amount of heat loss. Hence,

$$
Q_{l}=\frac{q}{V_{S}} S_{S}
$$

where, the heat loss $q$ is obtained through the contribution from conduction and radiation.

$$
q=h\left(T-T_{o}\right)+\varepsilon_{R} \sigma_{s}\left\{(T+273)^{4}-\left(T_{o}+273\right)^{4}\right\}
$$

Physical properties of titanium used in the calculation are shown in Table 1.

\section{Experimental Procedure}

A system of the cold crucible consists of a five-turned water cooled copper coil with $10 \mathrm{~mm}$ in diameter and a water cooled crucible made of copper with $45 \mathrm{~mm}$ in internal diameter, $65 \mathrm{~mm}$ in external diameter and 150 $\mathrm{mm}$ in height. A cylindrically straight crucible has 16 pieces of vertical slits periodically arranged along the circumferential direction in the middle height of the crucible. The width and the length of the slits are 1 and $55 \mathrm{~mm}$, respectively.

The maximum output of the high frequency generator used is $65 \mathrm{~kW}$ and the frequency is $20 \mathrm{kHz}$. The frequency was measued by the use of an oscilloscope. The inductance was evaluated from the resonance condition 
Table 1. Physical properties of $\mathrm{Ti}$ used in the calculation.

$\begin{array}{lcl}\text { Solidus temperature } & T_{S} & 1630^{\circ} \mathrm{C} \\ \text { Melting temperature } & T_{M} & 1645^{\circ} \mathrm{C} \\ \text { Liquidus temperature } & T_{L} & 1660^{\circ} \mathrm{C} \\ \text { Thermal conductivity } & \lambda & 21.7 \mathrm{~W} / \mathrm{m} \cdot{ }^{\circ} \mathrm{C} \\ \begin{array}{l}\text { Specific heat at room } \\ \text { temperature }\end{array} & C_{p} & 0.807 \mathrm{~kJ} / \mathrm{kg} \cdot{ }^{\circ} \mathrm{C} \\ \text { Latent heat during } & \Delta H & 418 \mathrm{~kJ} / \mathrm{kg} \\ \quad \text { solidification } & & \\ \text { Density } & \rho & 4510 \mathrm{~kg} / \mathrm{m}^{3} \\ \text { Electric conductivity } & \sigma & 0.688 \mathrm{MS} / \mathrm{m} \\ \text { Surface tension } & \alpha & 1.51 \mathrm{~N} / \mathrm{m} \\ \text { Kinematic viscosity in } & v_{L} & 1.6 \mu \mathrm{m}{ }^{2} / \mathrm{seC} \\ \quad \text { liquidus } & & \end{array}$

and the coil current was theoretically obtained through the reactance and the applied voltage.

The casting procedure is as follows. The hopper is filled with metallic raw material and then the hopper and the furnace chamber are vacuumed. A metallic primer with $44 \mathrm{~mm}$ in diameter is placed in a certain position in the crucible where the effect of an induction coil is active. Then hopper and furnace chamber are filled with argon with slight overpressure compared to atmospheric pressure. The power is gradually transmitted to the charge until it melts with the formation of a dome. The controlled supply of the raw material and the simultaneous continuous withdrawal of the ingot then be started.

Main items of measurements are as follows.

\section{(1) Solidification Front}

Tungsten powder is added on the melt as a tracer for finding the solidification front through the difference in darkness of the solidified structure.

\section{(2) Surface Velocity of the Melt}

Small size metallic material fed onto the dome floats on the melt surface with the metal flow for a few seconds on account of surface tension. The surface velocity was obtained through recording pictures of the tracer by a high speed video camera placed over the crucible, followed by making graphic analysis using the melt shape obtained through the electromagnetic field model 1. In the analysis, especially the materials floating along the normal direction to the alignment of feeder are chosen to minimize the error.

(3) Surface Temperature of the Melt

The surface temperature was measured by the use of radiation pyrometer in a contactless way.

\section{Validity of the Mathematical Model}

The validity of the electromagnetic field model 1 was already examined in the reference. ${ }^{4)}$ In the present study, the validity of the whole model was examined with respect to solidification front, surface velocity of the melt and the surface temperature of the melt. The standard calculation condition used in the comparison between the calculation and measurements is shown in Table 2. Parameters $P_{v}$ and $P_{H}$ were determined in such a way that $90 \%$ of the variation in the temperature dependence of viscosity and enthalpy is realized in the
Table 2. Standard calculation condition.

$\begin{array}{lcl}\text { Frequency of an applied AC field } & f & 20 \mathrm{kHz} \\ \text { Effective coil current } & J & 1290 \mathrm{~A} \\ \text { Melt volume above slit bottom } & V_{v} & 52 \mu \mathrm{m}^{3} \\ \text { Casting velocity } & v_{c} & 0 \mathrm{~m} / \mathrm{sec} \\ \text { Heat transfer coefficient } & h & 0.73 \mathrm{~kW} / \mathrm{m}^{2} \cdot{ }^{\circ} \mathrm{C} \\ \text { Emissivity } & \varepsilon_{R} & 0.15(-)) \\ \text { Kinematic viscosity in } & v_{s} & 160 \mu \mathrm{m}^{2} / \mathrm{sec} \\ \quad \text { solidus } & P_{v} & 5^{\circ} \mathrm{C} \\ \text { Parameter in the temperature } & & \\ \quad \text { dependence of viscosity } & & 50(-) \\ \text { Parameter in the temperature } & P_{H} & \\ \text { dependence of enthalpy } & & \end{array}$

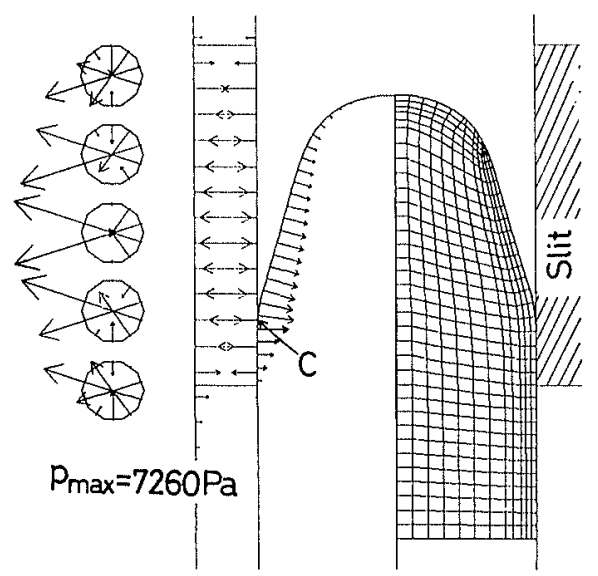

Fig. 3. Magnetic pressure working on the conductive sheet and the grids used in the calculation for the fields of velocity and temperatrue

temperature region between $T_{S}$ and $T_{L}$. The surface temperature of the melt obtained through the calculation was compared with that of experiment in the coil current between 1053 and $1580 \mathrm{~A}$.

\subsection{Calculated Results of the Electromagnetic Field Models 1 and 2}

The magnetic pressure working on the conductive sheet as well as the shape of the melt which were obtained through the electromagnetic field model 1 on standard calculation condition are shown in Fig. 3, together with the calculation grids for a charge used in the electromagnetic field model 2.

The magnetic pressure works as a pinching manner of the conductor. Especially, it is significant on the surface of the coil placed in the middle and close to the external surface of the crucible. Along the melt surface from the top to the bottom, the magnetic pressure increases approximately in proportional to the hydrostatic pressure of the melt to its maximum value at the point $\mathrm{C}$ where the surface of the melt and the wall of the crucible begin to touch, followed by the decrease. Grid spacing near the surface of the melt is arranged especially small to describe accurately the decay of the electromagnetic field in the bulk of a charge.

The contour lines of joule heat generation and the distribution of the magnetic body force in a charge is shown in Fig. 4. The joule heat generation and the electromagnetic body force manifest themselves the highest value at the point $\mathrm{C}$. They decrease dramatically 


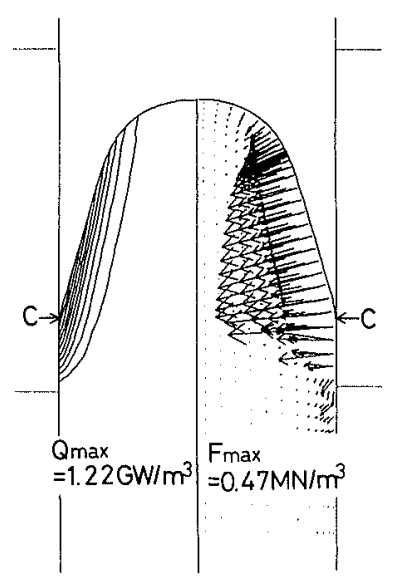

Fig. 4. Contour lines of joule heat generation and the distribution of magnetic body force in a charge.

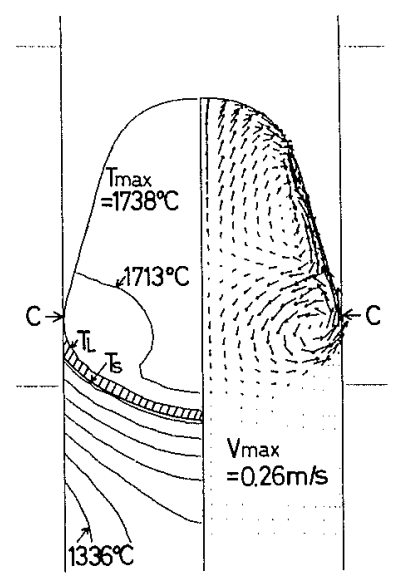

Fig. 5. Fields of temperature and velocity in a charge.

in the bulk along the normal direction to the surface because of the presence of skin effect, the depth of which is $4.3 \mathrm{~mm}$.

\subsection{Comparison of the Calculation with the Experiment}

The calculated fields of temperature and velocity in a charge on the standard calculation condition are shown in Fig. 5. Experimentally obtained solidification front at the beginning of the casting is shown in Fig. 6 .

The calculated solidification front was in agreement with the experimental one under the condition of the emissivity of 0.15 and the heat transfer coefficient between the charge and the crucible of $0.73 \mathrm{~kW} / \mathrm{m}^{2} \cdot{ }^{\circ} \mathrm{C}$.

The temperature of the melt pool is fairly homogeneous because of the mixing of fluid flow. On the surface of the charge a mushy zone is expected to appear near the point $\mathrm{C}$ where the charge begins to touch the wall. Two kinds of recirculation flow are expected to appear in the melt. The first one flows down along the melt surface from the dome top to the slightly upper point of $\mathrm{C}$ and returns through the bulk of the melt. The second one flows down in the bulk, followed by the flow up to the melt surface along the solidification front via point C. They make collision slightly above the point $\mathrm{C}$ in the vicinity of the surface. The maximum velocity of the melt is expected to be $0.26 \mathrm{~m} / \mathrm{sec}$ and the velocity reduces to the values between 0.04 and $0.2 \mathrm{~m} / \mathrm{sec}$ on the melt surface.

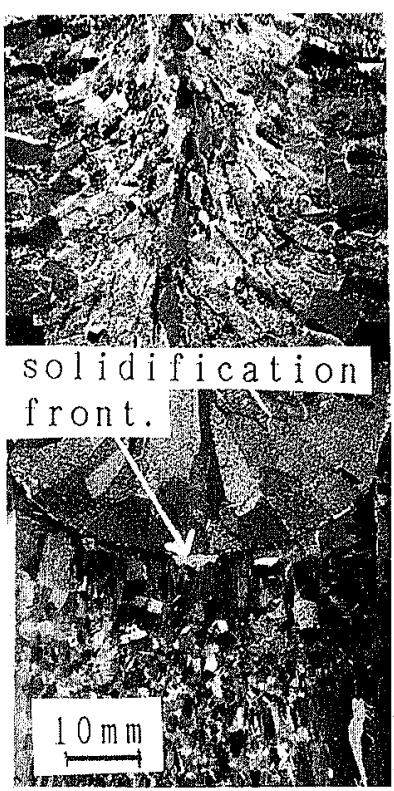

Fig. 6. Solidification front at the beginning of casting obtained by an addition of tungsten powder as a tracer.

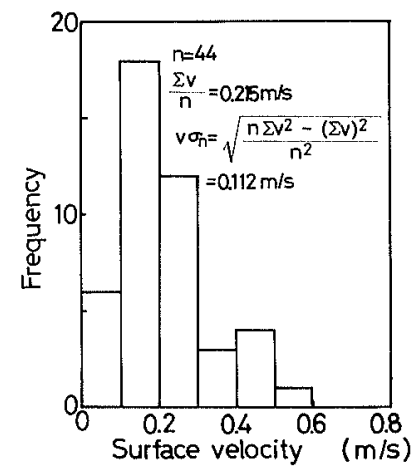

Fig. 7. Dispersion of the measured surface velocity of the melt.

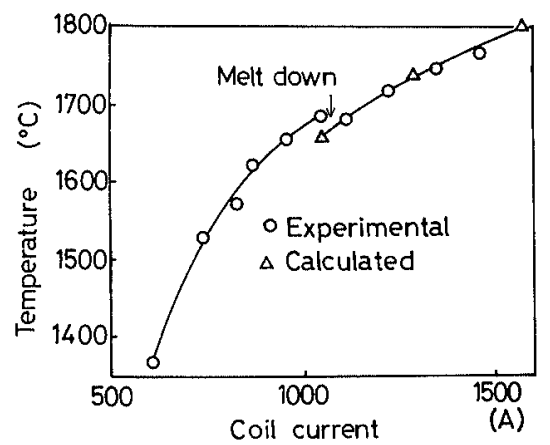

Fig. 8. Comparison of the calculated surface temperature with the experimental one in the variation of coil current.

The dispersion of the measured velocity on the melt surface is shown in Fig. 7. The mean velocity of the melt flow is $0.22 \mathrm{~m} / \mathrm{sec}$, which is slightly larger than the calculated one. In the measurement, the profile of the melt is not necessarily stationary because of the unsteady phenomena caused by the turbulence, such as bending or rotation of the dome shape. As a result, the measured value of the velocity is considered to become larger than the calculated one. Magnetic Reynolds number was estimated to be $5 \times 10^{-3}$ from the measurement of the surface velocity. 
A comparison of the calculated surface temperature of the melt with the measured one is shown in Fig. 8. The calculated temperature is in agreement with the measured one.

It is concluded from the comparison of the calculated results with the measured ones mentioned above that the validity of the mathematical model was confirmed through the measurements with respect to the profile of the solidification front, the surface temperature and the surface velocity.

\section{Calculated Results and Discussion}

In the following sections, the effects of the important operation parameters during continuous casting, such as coil current, dome height, heat loss and casting velocity, on the fields of fluid flow and the temperature were investigated by making use of the mathematical model.

\subsection{Coil Current}

In general, an increase in coil current resulted in the increase in the surface temperature of the charge. However, a small amount of abrupt decrease in the temperature was experimentally observed at the coil current of $1053 \mathrm{~A}$. The mechanism of this phenomena is considered to be as follows.

The shape of the charge changes itself from the cylindrical primer to dome when the charge is melt down. This leads to a weak electromagnetic interaction between the charge and the crucible accompanied with the increase in the amount of heat loss, since the distance between them increases and the melt is in touch with the wall. As a result, the joule heat generation decreases, followed by the progressing of solidification.

The relation between the coil current and the fields of the temperature and the velocity is shown in Fig. 9. Phenomena which is happening in a charge after being solidified at the coil current of $1053 \mathrm{~A}$ is considered to be the picture shown in the left hand side of Fig. 9. Namely, the temperature at the dome summit is low and the charge there is solidified. While the temperature is the highest at the region slightly above the point $\mathrm{C}$ and near the surface. Liquid metal flows with the slow velocity in a restricted region of the dome.

An increase in the coil current results in the elevation

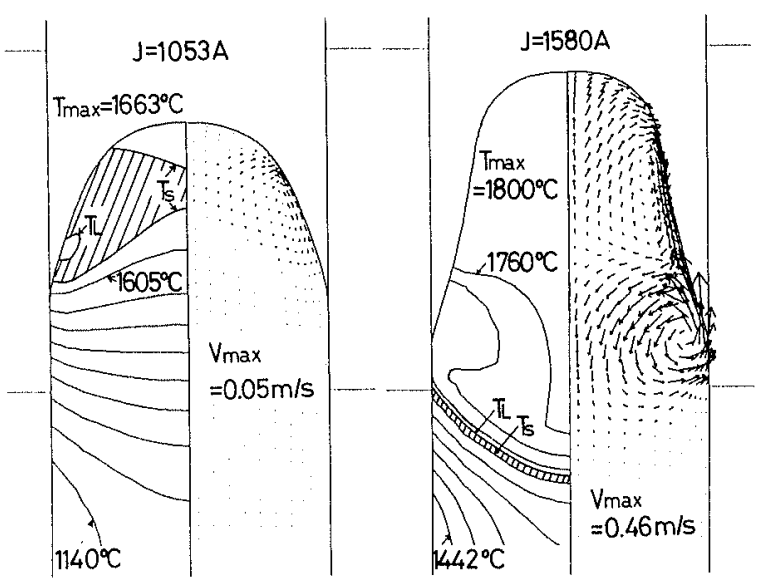

Fig. 9. Effect of coil current on the fields of temperature and velocity in a charge. of dome, the increase in the temperature, the increase in the fluid velocity and the descent of the solidification front, changing its shape from convex to concave.

In the low value of the coil current, the temperature of the charge is too low to melt the raw material being supplied. While, in the high value, it is sufficiently high to make continuous casting. However, since the melt is expected to be directly in touch with a certain part of the crucible wall, careful attention should be paid for the achievement of high efficient water cooling of the wall to avoid the melting of the wall. Hence, generally an appropriate value of the coil current is to be chosen depending on the casting condition, such as casting velocity, diameter of an ingot.

\subsection{Dome Height}

Figure 10 shows the effect of the volume of a charge above the slit bottom of the crucible, i.e. the effect of the dome height with respect to the coil arrangement, on the fields of temperature and velocity. With an increase in the volume, contact angle between the melt and the crucible wall increases accompanied with the deformation of the melt shape to the one similar to a cylinder with flat dome.

The maximum temperature of the melt is higher with the smaller volume in the range of the calculation. The amount of the joule heat generation transmitted from the coil into the melt above the slit bottom increases from 7.6 to $11.9 \mathrm{~kW}$ with an increase in the volume of the charge. However, the joule heat generation per unit volume decreases, because the volume of the charge increases from 36 to $68 \mu \mathrm{m}^{3}$, accompanied with the increases of surface area of the melt. As a result, the maximum temperature of the melt is low with the larger amount of volume of the charge.

In the experiment of the continuous casting, most of the raw material fed onto the dome summit is usually fine enough to be floated on the surface of the melt with the same velocity of the liquid metal because of the surface tension. In this case, most of the material is considered to be transported with the surface flow, followed by the entrance into the bulk of the melt through the region coming into collision with the two kinds of recirculation. It will experience the melting as well as

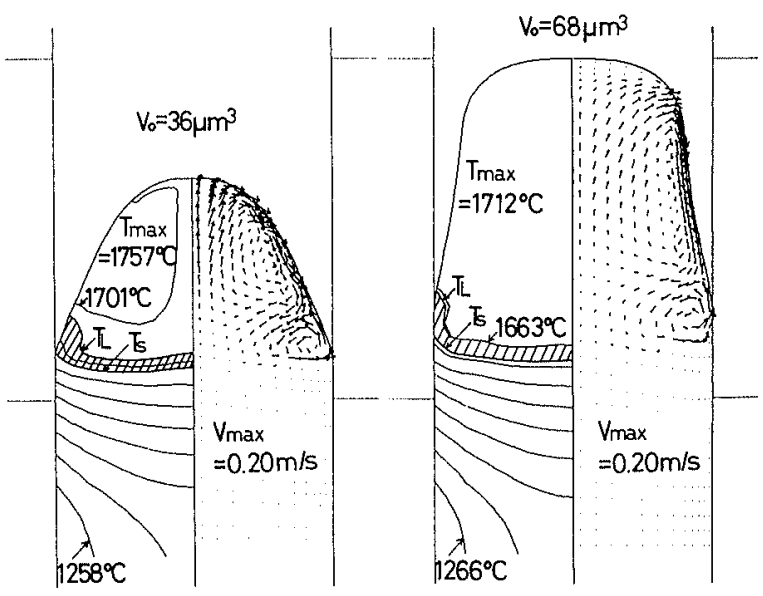

Fig. 10. Effect of charge volume above the slit bottom on the fields of temperature and velocity. 
the turbulent diffusion in the melt. A small part of the raw material with large size will descend through the first and the second recirculation flows to reach the solidification front where the temperature is too low to melt the material. To avoid the deposition of the unmelted raw material on the solidification front, it is necessary to make faster the recirculation velocity, deeper the melt pool and higher the melt temperature.

It is found from the comparison between Fig. 5 with $V_{o}=52 \mu \mathrm{m}^{3}$ and the left hand side of Fig. 10 with $V_{o}=36 \mu \mathrm{m}^{3}$ that in case of the low dome height, the velocity of the melt is slow and the pool depth is shallow, though the melt temperature is high. Especially, in case of the lower dome height with the smaller amount of melt volume than $36 \mu \mathrm{m}^{3}$, the temperature of the melt will become low, since the magnetic flux density works weakly on a charge. While, in case of the high dome height, the velocity of the melt becomes slow, the depth of the pool is shallow and the temperature of the melt is low, which is found from the comparison between Fig. 5 and the right hand side of Fig. 10 .

As a result, it is concluded from the calculation that an appropriate position of the dome height relative to the arrangement of coil is considered to exist to promote the melting of raw material.

\subsection{Heat Loss}

The effect of the heat transfer coefficient between the charge and the crucible wall on the fields of the temperature and the velocity is shown in Fig. 11. With an increase in the amount of the heat loss, the temperature of the melt is expected to decrease and the solidification front to ascend. Simultaneously, the velocity of the melt decreases and the region where liquid metal flows becomes small. This phenomena is significant when the heat transfer coefficient increases from 0.91 to $1.09 \mathrm{~kW} / \mathrm{m}^{2} \cdot{ }^{\circ} \mathrm{C}$. The reason is that the viscosity increases dramatically with the increase in the heat transfer coefficient, especially when the melt temperature is close to the solidification point.

The overall heat transfer coefficient including the effect of the radiation at the standard calculation condition is at most $0.73 \mathrm{KW} / \mathrm{m}^{2} \cdot{ }^{\circ} \mathrm{C}$. The value is approximately 0.08 times as small as that of the initial solidification of steel. ${ }^{7)}$ Hence, in a cold crucible, the contact between the charge and the wall is considered to be imperfect because of the presence of magnetic pressure.

\subsection{Casting Velocity}

The effect of the casting velocity on the fields of the temperature and the velocity is shown in Fig. 12. An increase in the casting velocity is expected to result in the decrease in the maximum temperature of the melt and the ascent of the solidification front. The velocity of the melt is hardly affected by the casting velocity in the range lower than $0.05 \mathrm{~mm} / \mathrm{sec}$.

However, beyond this value, the recirculation velocity of the melt becomes slow and the area of the second kind of recirculation is getting small. This is of disadvantage for the perfect melting of raw material, resulting the existence of the unmelted part of the material

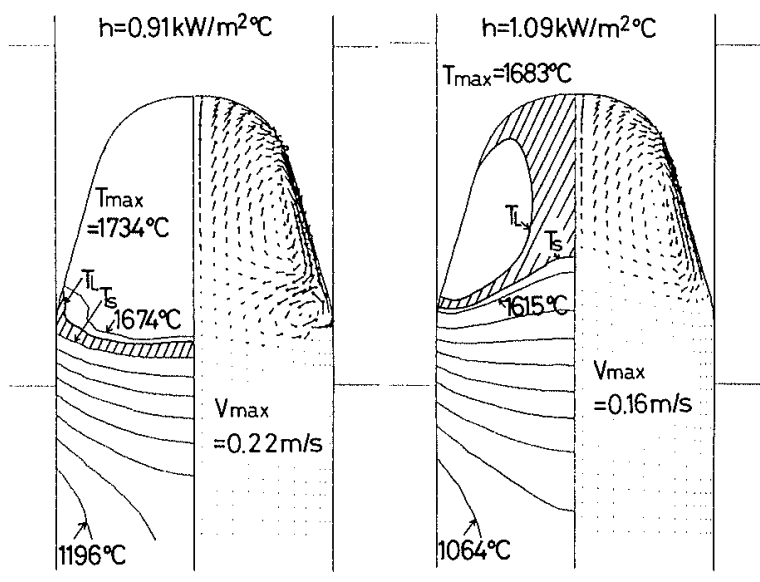

Fig. 11. Effect of heat transfer coefficient on the fields of temperature and velocity.

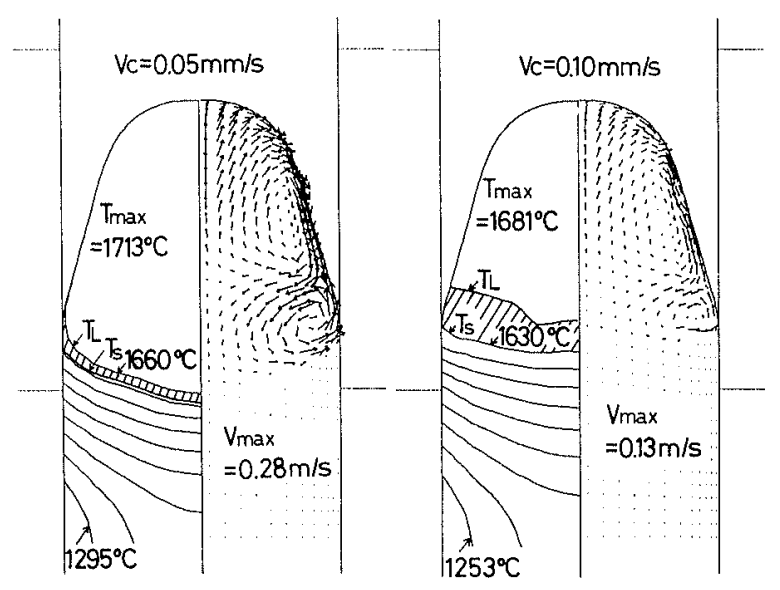

Fig. 12. Effect of casting velocity on the fields of temperature and velocity.

in the ingot. Hence, it is concluded from the calculation that a certain maximum value of casting velocity exists to promote the strong mixing of the pool. The maximum value of the casting velocity is dependent on the operation parameter, such as coil current and frequency. It is to be determined by the numerical calculation under the given operation parameter.

According to the useful information obtained through the numerical calculation, appropriate casting condition is chosen shown in Table 2 except for the casting velocity being $0.05 \mathrm{~mm} / \mathrm{sec}$. This operation condition of the continuous casting is considered to bring about the fields of the velocity and the temperature shown in the left hand side of Fig. 12. As a result, a titanium ingot shown in Fig. 6 was successfully obtained by making use of the continuous casting type of a cold crucible.

B. Maestrali et al. $^{3}$ ) predict that with an increase in the casting velocity, the solidification front on the axis of an ingot descends and that on the surface ascends. These are some differences from the present study in the results. A crucial decision must be awaited until a careful experiment is performed in the future.

\section{Conclusion}

An advanced mathematical model capable of describing melt flow coupled with heat transfer in a cold crucible 
confined by a free surface and a solidification front has been developed. The validity of the model was confirmed through measurements of the solidification front, the surface velocity and the surface temperature. Results obtained through the numerical calculations are summarized as follows.

(1) Generally, two kinds of recirculation flow exist in the melt, which make collision slightly above the contact point between the melt and the wall. As a result, the temperature of the melt is fairly homogeneous.

(2) An increase in the load relative to the power input, such as the increase in the heat loss, the increase in the casting velocity, the decrease in the coil current, results in the decrease in the melt temperature as well as the melt velocity and the ascent in the solidification front.

(3) An appropriate casting condition, such as the dome height with respect to the coil arrangement and the casting velocity, exists to promote the melting of metallic raw material.

(4) The casting experiment of titanium has successfully been conducted with the aid of the useful information obtained through the mathematical model, leading to the complete melt of the material.

(5) The heat transfer coefficient between the charge and the crucible is a tenth as small as that of the initial solidification of a steel, indicating the imperfect contact between them because of the presence of the electromagnetic pressure.

\section{Nomenclature}

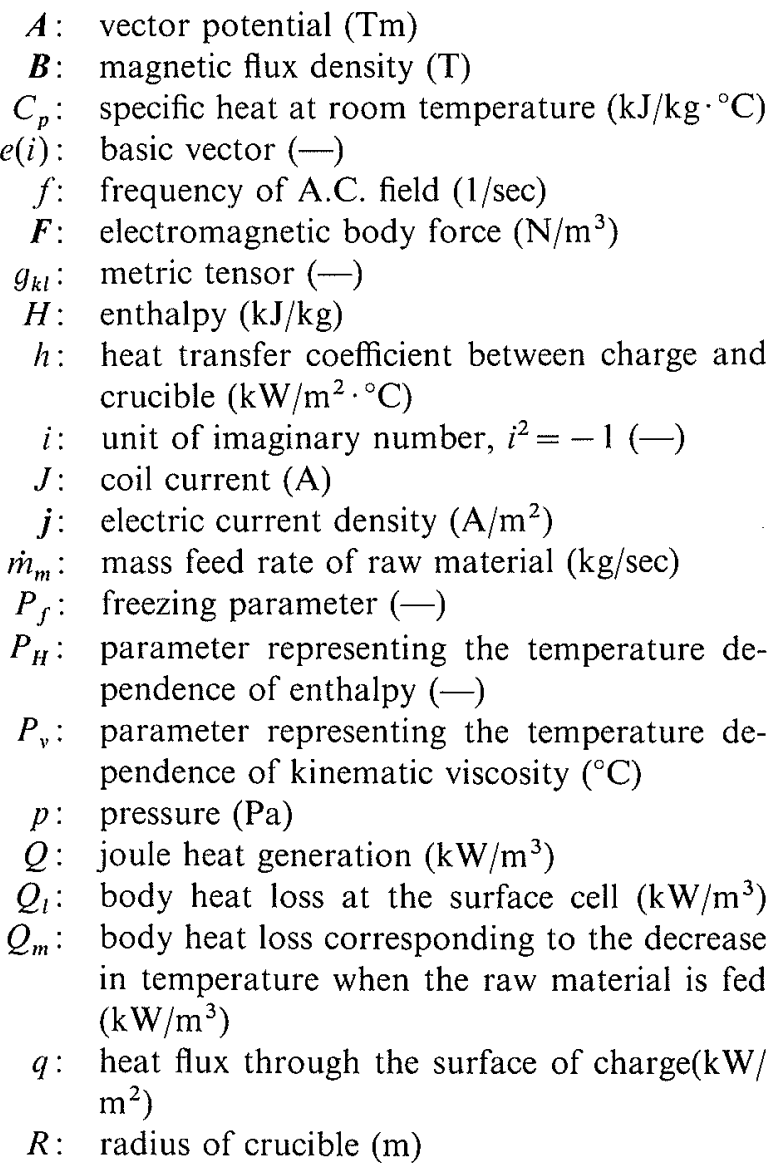

$r$ distance between observation point and integration point $(\mathrm{m})$

$S_{s}:$ area of surface cell $\left(\mathrm{m}^{2}\right)$

$T:$ temperature $\left({ }^{\circ} \mathrm{C}\right)$

$T_{L}$ : liquidus temperature $\left({ }^{\circ} \mathrm{C}\right)$

$T_{m}$ : temperature of material being fed $\left({ }^{\circ} \mathrm{C}\right)$

$T_{M}$ : melting temperature $=1 / 2\left(T_{L}+T_{S}\right)\left({ }^{\circ} \mathrm{C}\right)$

$\bar{T}_{p}$ : mean temperature of melt pool $\left({ }^{\circ} \mathrm{C}\right)$

$T_{S}:$ solidus temperature $\left({ }^{\circ} \mathrm{C}\right)$

$t:$ time $(\mathrm{sec})$

$V:$ volume $\left(\mathrm{m}^{3}\right)$

$V_{p}:$ volume of melt pool $\left(\mathrm{m}^{3}\right)$

$V_{o}:$ volume of charge above the slit bottom $\left(\mathrm{m}^{3}\right)$

$V_{s}:$ volume of surface cell $\left(\mathrm{m}^{3}\right)$

$\boldsymbol{v}=v(i):$ velocity vector $(\mathrm{m} / \mathrm{sec})$

$v_{c}$ : casting velocity $(\mathrm{m} / \mathrm{sec})$

$\boldsymbol{v}_{L}$ : velocity vector of liquid metal $(\mathrm{m} / \mathrm{sec})$

$x_{i}:$ coordinate $(\mathrm{m})$

$\Delta H$ : latent heat during solidification $(\mathrm{kJ} / \mathrm{kg})$

$\delta:$ skin depth $(\mathrm{m})$

$\varepsilon:$ divergence of velocity vector $(1 / \mathrm{sec})$

$\varepsilon_{H}$ : parameter representing the temperature dependence of enthalpy $\left({ }^{\circ} \mathrm{C}\right)$

$\varepsilon_{R}:$ emissivity of radiation $(-)$

$\theta$ : angle between calculation grid and casting direction (rad)

$\lambda:$ thermal conductivity $\left(\mathrm{kW} / \mathrm{m} \cdot{ }^{\circ} \mathrm{C}\right)$

$\mu$ : magnetic permeability $(\mathrm{H} / \mathrm{m})$

$v$ : kinematic viscosity $\left(\mathrm{m}^{2} / \mathrm{sec}\right)$

$v_{L}$ : kinematic viscosity of liquidus $\left(\mathrm{m}^{2} / \mathrm{sec}\right)$

$v_{S}$ : kinematic viscosity of solidus $\left(\mathrm{m}^{2} / \mathrm{sec}\right)$

$\xi^{i}$ : normal coordinate $(\mathrm{m})$

$\rho:$ mass density $\left(\mathrm{kg} / \mathrm{m}^{3}\right)$

$\sigma:$ electric conductivity $(\mathrm{S} / \mathrm{m})$

$\sigma_{S}:$ Stefan-Boltzmann factor $=5.67 \times 10^{-8}(\mathrm{~W} /$ $\left.\mathrm{m}^{2} \cdot{ }^{\circ} \mathrm{C}^{4}\right)$

$\phi: \quad$ scalar potential $(\mathrm{V})$

$\omega$ : angular frequency of $\mathrm{A}$. C. field $(\mathrm{rad} / \mathrm{sec})$

\section{REFERENCES}

1) P. Paillere, J. F. Wadier, Y. Boussant-Roux and M. Garnier: Proc. Sixth World Conf. on Titanium, Société Française de Métallurgie, Les Ulis Cedex, France, (1988), 583.

2) K. Kaneko, T. Misawa and K. Tabata: Proc. of the Sixth IISC, Vol. 4, ISIJ, Tokyo, (1990), 254.

3) B. Maestrali, Ph. Masse and A. Gagnoud: Workshop "Control of Free Surface of Liquid Metals by a Magnetic Field: Modeling, Analysis and Applications", Institute National Polytechnique de Larraine, Université de NANCY, INRIA Lorraine-C.N.R.S., Nancy, (1990).

4) T. Tanaka, K. Kurita and A. Kuroda: ISIJ Int., 31, (1991), 350.

5) N. El-Kaddah and F. A. Acosta-Gonzalez: Proc. of an Int. Symp. on Casting of Near Net Shape Products, The Metallurgical Soc., Inc., Hawaii, (1988), 423

6) I. S. Sokolnikoff: Tensor Analysis , John Wiley \& Sons, Inc., New York, (1964), 351.

7) H. Ichihashi, M. Yoshihara, Y. Shirai, Y. Sugitani and N. Yoshida: Proc. of Japan-U.S. Cooperative Science Program Seminar on Solidification Processing of Advanced Materials, Oiso, (1989), 263. 